\title{
Percepciones de estudiantes universitarios chilenos sobre uso de redes sociales y trabajo colaborativo
}

\section{(Perceptions of Chilean university students about the use of social networks and collaborative work)}

\author{
Julio Cabero-Almenara \\ Universidad de Sevilla (España) \\ Annachiara Del Prete \\ María Luisa Arancibia Muñoz \\ Universidad Tecnológica de Chile, INACAP (Chile)
}

DOI: http://dx.doi.org/10.5944/ried.22.2.22847

\section{Cómo referenciar este artículo:}

Cabero-Almerara, J., Del Prete, A., y Arancibia, M. L. (2019). Percepciones de estudiantes universitarios chilenos sobre uso de redes sociales y trabajo colaborativo RIED. Revista Iberoamericana de Educación a Distancia, 22(2), pp. 35-55. doi: http://dx.doi.org/10.5944/ried.22.2.22847

\section{Resumen}

En los últimos años hemos sido testigos de un uso, siempre más difundido, de las redes sociales digitales (RSD), con fines educativos. El alumnado declara hacer un uso didáctico de las redes y sus herramientas y, al mismo tiempo, reconoce el trabajo colaborativo como una metodología que promueve su aprendizaje y que viene propiciada por el uso de las redes sociales. Entre los propósitos del siguiente estudio, se encuentra indagar las percepciones que el alumnado tiene respecto al uso de las redes sociales como herramienta educativa y conocer sus preferencias con respecto al trabajo colaborativo. La investigación realizada se encuadra dentro de las denominadas ex post facto, no experimental y descriptiva. La muestra de la investigación estuvo formada por 460 estudiantes de diferentes carreras del área de ingeniería de una institución de educación superior chilena, durante el curso académico 2017-18. Entre los resultados destacables, podemos afirmar que Internet se está presentando como una de las tecnologías que va penetrando con más fuerza en las universidades, y que los alumnos la suelen utilizar constantemente para fines educativos. Es por estas razones que consideramos que existen las condiciones iniciales para la incorporación de las redes sociales a la enseñanza formal. Además, nuestros resultados muestran que el interés de trabajar con otros estudiantes determina la actitud del alumnado hacia el trabajo colaborativo. Ello nos lleva a reclamar la 
necesidad de que el docente se asegure de las percepciones que tienen los alumnos, y aplique estrategias específicas.

Palabras clave: redes sociales; trabajo colaborativo; educación superior.

\begin{abstract}
In recent years we have witnessed an increasingly widespread use of social digital networks (DSN) for educational purposes. The students declare to make a didactic use of the networks and their tools and, at the same time, they recognize the collaborative work as a methodology that promotes their learning and that is fostered by the use of the DSN. Among the purposes of the following study, we find the question of the perceptions that students have regarding the use of social networks as an educational tool and to know their preferences regarding collaborative work. The research carried out falls within the so-called "ex post facto", not experimental and descriptive. The sample of the research was formed by 460 students who studied different degrees in the engineering area of a Chilean higher education institution, during the 2017-18 academic year. Among the remarkable results, we can affirm that the Internet is presenting itself as one of the technologies that is penetrating more strongly in the Universities, and that the students use it constantly for educational purposes. It is for these reasons that we consider that there are initial conditions for the incorporation of social networks to formal education. In addition, our results show that the interest to work with other students determines the attitude of students towards collaborative work. This leads us to claim the need for the teacher to ensure the perceptions that students have, and apply specific strategies.
\end{abstract}

Keywords: social networks; collaborative work; higher education.

Las redes sociales digitales (RSD) son unas de las tecnologías que han impulsado de manera significativa el uso de Internet entre los jóvenes y adolescentes y, aunque su utilización se asocia al ocio (Sanz, Alonso, Sáenz, Ponce y Valdemoros, 2018), también ha despertado un gran interés educativo y didáctico, en su aplicación en la enseñanza universitaria (Abramson, 2011). Asociadas a las RSD se encuentran las diferentes herramientas de la web 2.o, que han potenciado el desarrollo y aplicación del denominado software social (Castaño, 2012), que puede ser entendido de acuerdo con Parameswaran \& Whinston (2007) como una categoría de aplicaciones y servicios que facilitan la acción colectiva y la interacción social en línea, con un rico intercambio de información multimedia, la cual genera una evolución del conocimiento de manera general.

Las definiciones que se han realizado de las RSD son diversas, pero, de manera general, coinciden en asumir que son un lugar, donde un grupo de personas se relacionan, comparten información, se comunican e interaccionan entre sí y crean comunidades, todo esto en red. Lo verdaderamente significativo de este tipo de 
redes es el nuevo modelo de comunicación y de relaciones que establecen entre los comunicantes, sirviendo como elemento para la democratización de la información y la creación de proconsumidores (Della-Porta, 2015).

\section{LA REDES SOCIALES Y LA EDUCACIÓN}

Dentro de las RSD existen una diversidad de tipos, en función de su grado de abertura, su temática, el que sean coordinadas o no, etc. (De Haro, 2011). Pero lo que aquí interesa es que, en los últimos tiempos, están alcanzado un fuerte uso en la educación, siendo utilizadas para diversos aspectos, a saber, medio alternativo de comunicación, aprendizaje de idiomas, plataforma o LMS (en inglés, Learning management system) para la formación virtual, socialización de los estudiantes, potenciación dela educación inclusiva, mejorar la comunicación y la relación profesorestudiante y profesor-padre de estudiantes, facilitar el contacto constante docente y discente. Como medio alternativo de comunicación entre los participantes en la acción formativa fomentan los aprendizajes formales y no formales, para la tutoría y asesoría de estudiantes y padres, para la realización de trabajos colaborativos entre los estudiantes (Vázquez y Cabero, 2015; Cruz, 2016; Fromet, García y Bohórquez, 2017; Delgado-García, García-Prieto y Gómez-Hurtado, 2018) y para el uso que aquí preocupa: el trabajo colaborativo virtual (Cabero, 2014).

Al mismo tiempo se están señalando diferentes aspectos a tener en cuenta para su incorporación en la educación, tales como el riesgo de privacidad, sobre todo, en las redes abiertas, la novedad del medio, el imaginario social de que es posible una traslación directa desde el uso de ocio y particular al educativo. No todas las personas muestran la misma actitud en el trabajo mediante redes, y la falta de formación del docente y discente para su incorporación a la enseñanza (Vázquez y Cabero, 2015; Fromet, García y Bohórquez, 2017; Alter, 2018; García, Tirado y Hernando, 2018).

En el ámbito de la formación universitaria las redes sociales digitales se están integrando como herramientas significativas (Guzmán, Rueda y Mendoza, 2017) y, respecto a ellas, se están desarrollando, en los últimos tiempos, diferentes investigaciones que están poniendo de manifiesto como su uso favorece la interacción docente discente, y mejora el rendimiento alcanzado por los estudiantes (Chávez y Gutiérrez, 2015; Romero y Garay, 2017; Tejada, Garay y Romero, 2017). Si bien existen estudios (Roblyer et al., 2010), quien indica que los profesores y los estudiantes difieren ligeramente en los usos actuales y futuros de las redes sociales, percibiéndose más como herramienta social que educativa, y siendo los estudiantes los que se muestran más atentos a su utilización en los estudios que los docentes. Por su parte, López, Flores, Espinoza y Rojo (2017) sostienen que, con una dirección y un diseño adecuados, las redes sociales, en el caso concreto de su estudio centrado en Facebook, pueden propiciar ambientes de aprendizaje dinámicos y atractivos para los estudiantes de educación superior. 
En este contexto, Artola, Gálvez y González (2018), en su investigación, analizan la preferencia demostrada por los estudiantes por el uso educativo de las RSD. Los autores identifican tres grupos de usuarios de RSD para el aprendizaje: 1) usuarios básicos (formado por 15.08\% de la muestra, estudiantes que consideran que las tareas realizadas en grupo contribuyen poco o nada a su formación y las clases teóricas y el trabajo individual son los métodos que más contribuyen a su formación), 2) usuarios moderados (agrupa el 29.3\% del alumnado, estudiantes que declaran que los ejercicios en el aula contribuyen bastante a su formación), y 3) usuarios intensivos (contiene al 55.59\% de los estudiantes y que consideran que los ejercicios realizados en el aula hacen una contribución muy importante a su formación académica y que valoran muy positivamente la contribución de otros métodos como las clases prácticas). En síntesis:

han identificado tres grupos claramente diferenciados. Por un lado, el grupo de usuarios intensivos de estas herramientas, que constituye más de la mitad del alumnado; por otro, el grupo de usuarios básicos (15\% del alumnado), que parece mostrar preferencia por las formas más tradicionales de enseñanza-aprendizaje (clases teóricas y trabajo individual) y muestra un interés muy bajo en el uso de redes sociales, por el grupo de alumnos que se manifiesta como usuarios moderados. (Artola, Gálvez y González, 2018, p. 31).

Ahora bien, es importante destacar que la significación educativa de las RSD viene determinada por una planificación específica por parte de los docentes y por la realización de actividades de iniciación destinadas a que los estudiantes aprendan su uso educativo (Gavilán, Martínez y Fernández, 2017). Como apuntan Delgado-García, García-Prieto y Gómez-Hurtado (2018) tras un estudio realizado, su utilización exitosa, como herramienta en el aprendizaje del alumnado, requiere la necesidad de un cambio en las estructuras y la formación docente para mejorar la calidad de la enseñanza.

\section{LAS REDES SOCIALES COMO ENTORNOS PARA EL APRENDIZAJE COLABORATIVO}

Centrándonos en la otra variable de nuestro estudio, el trabajo colaborativo en redes, cabe señalar que esta es una estrategia y metodología de instrucción basada en la creencia de que el aprendizaje se incrementa cuando los estudiantes desarrollan destrezas cooperativas para aprender y solucionar los problemas y acciones educativas en las cuales se ven inmersos de forma conjunta. Como señala Cabero (2014), el trabajo colaborativo es una estrategia que está basada en la relación de interdependencia de todos los estudiantes que trabajan en la acción formativa, debe existir una fuerte responsabilidad individual de todos en la acción, debe existir una fuerte interacción, también entre todos, y asumir que el aprendizaje no se consigue 
solo a partir de la interacción, sino más bien por la justificación de las decisiones adoptadas, los esfuerzos realizados para resolver los problemas y la co-construcción de significados por todos los miembros.

Para Levis (2011), las RSD, como entornos para el aprendizaje colaborativo, ofrecen una serie de posibilidades, tales como propiciar actividades grupales, facilitar el trabajo interdisciplinar, fomentan las relaciones horizontales entre docentes y discentes, potencian dinámicas colaborativas y cooperativas de estudio y de investigación, impulsan la producción colectiva de conocimiento, ofrecen una plataforma de comunicación versátil que permite distintos modos de comunicación interpersonal en línea (pública y/o privada, y sincrónica o asincrónica), acentúan el sentido de pertenencia al grupo, favorecen la creación de comunidades de enseñanza y aprendizaje, posibilitan que se desarrollen modelos de enseñanza y aprendizaje no sustentadas en posiciones jerárquicas, contribuyen a un modelo de enseñanza activo donde los estudiantes comparten información y documentos en diferentes formatos sobre temas de interés para ellos, y favorecen un mejor uso de los conocimientos previos, los intereses, la curiosidad y la capacidad de exploración de los estudiantes. A estos aspectos se les podría incorporar, como señalan García-Valcarcel et al. (2012), promover comportamientos prosociales entre los participantes.

Ahora bien, para que la aplicación de técnicas de trabajo colaborativo a través de redes, sea significativa, deben tenerse en cuenta una serie de variables, que van desde la planificación de la acción por parte del docente, la claridad de las normas que le sean indicadas a los estudiantes y el número de miembros de cada grupo (AbuSeileek, 2012; Jahang et al., 2012). Dentro de estos aspectos, no debemos olvidar la actitud y creencias que el alumno tenga de esta estrategia y, por tanto, de sus ganas de trabajar en equipo y de forma colaborativa (Baleghizadeh, 20l2). Con respecto a estos últimos aspectos, por lo general, muestran actitudes positivas y significativas ( $\mathrm{Ku}$, Tseng, Akarasriwo., 2013; Cabero, 2014), aunque también aparecen trabajos que expresan que los alumnos tienen una falta de cultura de colaboración y prefieren trabajar de forma individual (Cabero y Marín, 2014).

Por lo que se refiere a los resultados respecto a la preferencia hacia el trabajo colaborativo y su relación con el género de los estudiantes, tenemos que señalar que han sido contradictorios tendiendo, por lo general, a no encontrar diferencias, aunque, en algunos casos, sí se han encontrado una actitud más positiva hacia las mismas por parte de las mujeres (Cabero, Barroso, Llorente y Yáñez, 2016).

\section{METODOLOGÍA}

La investigación realizada se enmarca dentro de las denominadas ex post facto, no experimental y descriptiva, por tanto, es un tipo de investigación que plantea la validación de las hipótesis cuando el fenómeno ya ha sucedido (Bisquerra, 2004), o se encuentra en fase de desarrollo, y en las cuales el investigador no manipula ninguna variable experimental. 
En su puesta en acción se siguieron una serie de fases:

a. Selección del instrumento de diagnóstico.

b. Adaptación del instrumento al contexto chileno.

c. Identificación de la muestra.

d. Creación y administración electrónica del instrumento.

e. Fiabilización del instrumento.

f. Análisis de resultados y elaboración de conclusiones.

\section{Objetivos de la investigación}

En nuestra investigación se persiguen los siguientes objetivos:

- Indagar las percepciones que los alumnos tienen respecto al software social.

- Conocer las preferencias que los estudiantes presentan respecto al trabajo en grupo, colaborativo y respecto al trabajo individual.

- Analizar si existen diferencias significativas en función del género de los estudiantes, en cuanto a las percepciones que tienen en relación al trabajo en grupo e individual.

- Conocer si existen diferencias significativas de las percepciones de los estudiantes en relación al trabajo en grupo e individual, en función de su edad.

- Determinar qué herramientas de software social utilizan preferentemente.

- Determinar si el uso de las herramientas de software social depende de la edad, y el género de los estudiantes.

Al mismo tiempo se perseguía validar para el contexto chileno el instrumento "Social Software survey used with unpaced undergrad" elaborado por Anderson et al. (2009), y ya utilizado en el contexto latinoamericano por Cabero (2014).

\section{La muestra}

La muestra de la investigación estuvo formada por 460 estudiantes de diferentes grados y que cursaban diferentes carreras del área de ingeniería (automotriz, maquinaria pesada, química, construcción, industrial y eléctrica) en la sede de Renca de la Universidad Tecnológica de Chile INACAP, durante el curso académico 201718. La Universidad Tecnológica de Chile (UTC) INACAP es la segunda universidad de mayor tamaño del país en matrícula de pregrado, con aproximadamente 35 mil estudiantes. En su rol de organismo técnico de capacitación -OTEC-, INACAP es la universidad con mayor nivel de actividad en programas de capacitación, y la única con presencia en las quince regiones del país. La Universidad conforma junto a la Corporación INACAP, el Instituto Profesional (IT) INACAP y el Centro de Formación Técnica (CFT) INACAP, el Sistema Integrado INACAP, sistema de 
educación superior único en Chile, que forma, en las 12 áreas formativas, a más de 120 mil estudiantes a nivel nacional y cuenta con más de ocho mil colaboradores, académicos y administrativos.

Por el tipo de muestreo, podemos considerar la investigación del tipo no probabilístico (Sabariego, 2012), que viene determinada por la facilidad de acceso que el investigador tiene a los sujetos de estudio.

De los estudiantes participantes en la presente experimentación, 356 (77,39\%) eran hombres y $104(22,61 \%)$ mujeres; de la totalidad de la muestra, $242(52,61 \%)$ tenían una edad comprendida entres 17 a 20 años, 118 (25,65\%) de entre 21 a 24 años, 48 (10,43\%) entre 25 y 28 años, y 52 (11,30\%) más de 28 años de edad. Para la descripción de la muestra de estudio consideramos interesante señalar que 332 estudiantes $(72,17 \%)$ indicaron que habían realizado acciones formativas a través de Internet a lo largo de sus estudios universitarios, mientras $128(27,83 \%)$ declararon que no. Al mismo tiempo, encontramos que 410 estudiantes $(89,13 \%)$ declaran que sí acceden de forma regular a Internet con un fin educativo frente a 50 casos $(10,87 \%)$ que no lo hacen.

\section{Instrumento de recogida de información}

El instrumento utilizado para la recogida de datos estuvo fundamentado en la adaptación que se realizó del cuestionario "Social Software survey used with unpaced undergrad" (Anderson et al., 2009), que realizó Cabero (2014) en diferentes universidades españolas y latinoamericanas. El instrumento utilizado en la presente investigación quedó conformado por 71 ítems, que analizaban diferentes dimensiones, a saber, aspectos generales destinados a la caracterización de la muestra (género, edad, modalidad de estudio cursado, entre otros) (9 ítems), preferencia para trabajar en grupo o de forma individual (27 ítems), habilidades técnicas tecnológicas (15 ítems), experiencia en software social (10 ítems), y uso de distintas herramientas de software social (10 ítems). La estructura de las preguntas del cuestionario era tipo Likert y se administró vía internet.

Su índice de fiabilidad se obtuvo mediante la alfa de Crombach, procedimiento sugerido para este tipo de instrumento por diferentes autores ( $\mathrm{O}^{\prime}$ Dwyer y Bernauer, 2014), alcanzándose los valores presentados en la tabla 1.

Tabla 1. Alfa de Crombach

\begin{tabular}{|l|l|}
\hline & \multicolumn{1}{|c|}{$\boldsymbol{A l f a}$} \\
\hline Total del instrumento & $\mathbf{0 , 8 3 8}$ \\
\hline Preferencia de aprendizaje & $\mathbf{0 , 7 4 7}$ \\
\hline Habilidades técnicas-tecnológicas & $\mathbf{0 , 9 1 7}$ \\
\hline Experiencia en software social & $\mathbf{0 , 7 7 8}$ \\
\hline Software social para el aprendizaje & $\mathbf{0 , 8 7 4}$ \\
\hline
\end{tabular}


Correlaciones que pueden considerarse "altas" (Mateo, 2006), y sugieren altos niveles de fiabilidad del instrumento. Señalar que los valores fueron similares a los obtenidos por Anderson (2009) y Cabero (2014).

Finalmente, indicar que también obtuvimos la correlación ítem total, por si la eliminación de algún ítem implicaba el aumento de los valores de fiabilidad, pero los valores encontrados no lo sugerían.

\section{RESULTADOS}

En primer lugar, se señala que la gran mayoría de encuestados indicaron que sí habían realizado acciones formativas a través de Internet a lo largo de sus estudios universitarios ( $\mathrm{f}=332,72,17 \%)$, y que solían acceder de forma regular a Internet con fines educativos $(\mathrm{f}=410,89,13 \%)$.

Por lo que se refiere a su experiencia en el trabajo en grupo, la mayoría de los estudiantes señalaron que había sido "positiva" ( $\mathrm{f}=\mathbf{2 4 1}, 53,32 \%)$ o "muy positiva" $(\mathrm{f}=114,25,22 \%)$, siendo las respuestas referidas a una valoración negativa, muy bajas ("muy negativa" $\mathrm{f}=86,19,03 \%$ y "negativa" $\mathrm{f}=11,2,43 \%$ ).

Respecto al grado de interés que los estudiantes mostraban por llevar a cabo acciones de colaboración con otros estudiantes de su curso, las opciones de respuestas con más porcentajes fueron: "interesado" ( $\mathrm{f}=\mathbf{2 4 5}, 53,38 \%)$ y "muy interesado $(\mathrm{f}=\mathbf{1 2 7}$, 27,67\%); indicar que solo el 10,02 ( $\mathrm{f}=46$ ) señaló que "no lo sabía”.

Por lo que se refiere a las redes sociales que fundamentalmente utilizaban, en la tabla 2 se presentan los resultados alcanzados, donde podríamos destacar el bajo número de estudiantes que indicaron que no utilizaban ninguna red social $(\mathrm{f}=39$, $8,63 \%)$.

Tabla 2. Redes sociales utilizadas por los estudiantes

\begin{tabular}{|l|c|c|}
\hline \multicolumn{1}{|c|}{ Red Social } & $\boldsymbol{f}$ & $\boldsymbol{\%}$ \\
\hline Facebook & 302 & $66,81 \%$ \\
\hline Twitter & 59 & $13,05 \%$ \\
\hline Linkedin & 33 & $7,30 \%$ \\
\hline Hi5 & 19 & $4,20 \%$ \\
\hline Ninguna & 39 & $8,63 \%$ \\
\hline
\end{tabular}

En el análisis de las medias y desviaciones típicas alcanzadas por los estudiantes en cada una de las dimensiones e ítems que conformaban el instrumento, observamos que todos los casos tienden a centrarse en valores ligeramente superiores a los centrales ofrecidos en las escalas. Por tanto, se puede señalar que los alumnos tendieron a mostrarse de acuerdo con los diferentes indicadores propuestos. En particular, por lo que se refiere a la dimensión "preferencias de aprendizaje", las 
puntuaciones medias que alcanzaron en los diferentes ítems propuestos y en la totalidad del instrumento, se presentan en la tabla 3.

Tabla 3. Preferencias de aprendizaje

\begin{tabular}{|l|c|c|}
\hline & M & D.t. \\
\hline Trabajar en un grupo conlleva obtener resultados negativos. & 2,09 &, 99 \\
\hline $\begin{array}{l}\text { Un profesor puede ayudar a sus estudiantes cuando están trabajando en } \\
\text { grupo. }\end{array}$ & 3,89 &, 80 \\
\hline Prefiero trabajar de forma individual para así poder avanzar rápidamente. & 3,04 &, 95 \\
\hline $\begin{array}{l}\text { El trabajo en grupo es útil para reunir las ideas de todos y tomar una } \\
\text { decisión. }\end{array}$ & 3,91 &, 78 \\
\hline $\begin{array}{l}\text { Cuando un grupo o clase necesita hacer algo importante, me ayuda más } \\
\text { hacer el trabajo en grupo que solo por mi cuenta. }\end{array}$ & 3,46 &, 90 \\
\hline Trabajar en un grupo me da miedo. & 1,60 &, 89 \\
\hline No me gusta trabajar solo. & 2,74 & 1,03 \\
\hline En una discusión de grupo, nunca se llegan a conclusiones importantes. & 2,39 & 1,05 \\
\hline Me gusta que las clases se desarrollen a través del trabajo en grupo. & 3,45 &, 87 \\
\hline $\begin{array}{l}\text { Me gusta ser capaz de utilizar las ideas de otras personas, así como las mías } \\
\text { propias. }\end{array}$ & 3,62 &, 87 \\
\hline $\begin{array}{l}\text { Si trabajo por mí mismo la mayor parte del tiempo, me vuelvo solitario y me } \\
\text { encuentro infeliz. }\end{array}$ & 2,32 & 1,11 \\
\hline Los trabajos se hacen más rápidos si todos colaboramos. & 3,93 &, 78 \\
\hline Mi trabajo es de mayor calidad si lo hago solo. & 2,94 &, 91 \\
\hline Me gusta ayudar a otras personas al trabajar en grupo. & 3,86 &, 77 \\
\hline Si trabajo por mi cuenta sabré desenvolverme mejor en el futuro. & 3,23 &, 99 \\
\hline No sé trabajar solo. & 1,82 &, 94 \\
\hline Me gusta más mi trabajo cuando lo hago yo solo sin ayuda de nadie. & 2,83 &, 93 \\
\hline Otros estudiantes no necesitan saber lo que hago cuando estoy estudiando. & 2,83 & 1,04 \\
\hline $\begin{array}{l}\text { Trabajar en grupo ahora me ayudará a trabajar con otras personas en el } \\
\text { futuro. }\end{array}$ & 3,91 &, 75 \\
\hline Me gusta mantener mis ideas. & 3,63 &, 80 \\
\hline $\begin{array}{l}\text { El profesor puede ayudar a seleccionar el trabajo más adecuado para cada } \\
\text { estudiante. }\end{array}$ & 3,49 &, 89 \\
\hline El trabajo con otros estudiantes me puede ayudar a aprender. & 3,88 &, 69 \\
\hline Me gusta trabajar por mi cuenta sin prestar atención a otros compañeros. & 2,35 & 1,01 \\
\hline No me gusta trabajar con otros compañeros. & 2,02 & 1,03 \\
\hline "Preferencias de aprendizaje” & $\mathbf{3 , 0 5}$ &, 35 \\
\hline
\end{tabular}

Nota: escala De $1=$ totalmente en desacuerdo a 5 = totalmente de acuerdo 
El análisis de las puntuaciones medias alcanzadas permite señalar el acuerdo mostrado por los estudiantes por el trabajo en grupo: "Los trabajos se hacen más rápidos si todos colaboramos" (3,93), "El trabajo en grupo es útil para reunir las ideas de todos y tomar una decisión" (3,91), "Trabajar en grupo ahora me ayudará a trabajar con otras personas en el futuro" $(3,91)$ o "Trabajar en un grupo me da miedo" $(1,60)$.

Respecto a la dimensión habilidades técnicas-tecnológicas, se presentan, a continuación, las puntuaciones alcanzadas.

Tabla 4. Habilidades Técnicas-Tecnológicas

\begin{tabular}{|l|c|c|}
\hline & M & D.tp. \\
\hline Me gusta el uso de los computadores para la investigación y la educación. & 3,97 &, 80 \\
\hline $\begin{array}{l}\text { Me gusta comunicarme con los demás mediante la comunicación asistida } \\
\text { por computadores (correo electrónico, mensajes de texto) como apoyo a mi } \\
\text { aprendizaje. }\end{array}$ & 3,67 &, 89 \\
\hline Paso mucho tiempo en Internet. & 3,55 &, 90 \\
\hline $\begin{array}{l}\text { Sé cómo enviar y recibir mensajes y archivos adjuntos a través de } \\
\text { diversas herramientas de comunicación (correo electrónico, mensajería } \\
\text { instantánea,...). }\end{array}$ & 3,99 &, 77 \\
\hline $\begin{array}{l}\text { Mi computador es seguro ante las amenazas que puedan surgir cuando me } \\
\text { conecto a Internet. }\end{array}$ & 3,53 &, 95 \\
\hline $\begin{array}{l}\text { Soy bueno para encontrar lo que busco cuando utilizo los motores de } \\
\text { búsqueda de Internet (Google, Yahoo,...). }\end{array}$ & 3,87 &, 77 \\
\hline $\begin{array}{l}\text { Cuando se confunden mis compañeros sobre cómo hacer algo con el } \\
\text { computador, yo estoy capacitado para buscar la información para resolver el } \\
\text { problema (función de ayuda, documentación,...). }\end{array}$ & 3,59 &, 87 \\
\hline $\begin{array}{l}\text { Estoy capacitado para escribir documentos a través de procesadores de texto } \\
\text { (por ejemplo utilizando el subrayado, las negrillas,...). }\end{array}$ & 3,85 &, 87 \\
\hline Sé cómo instalar software para apoyar mi aprendizaje con el computador & 3,52 & $\mathbf{1 , 0 0}$ \\
\hline Me siento a gusto al trabajar con computadores. & 3,91 &, 80 \\
\hline $\begin{array}{l}\text { Puedo solucionar la mayoría de los problemas asociados al uso de un } \\
\text { computador. }\end{array}$ & 3,51 &, 93 \\
\hline Tengo amplia experiencia en el uso de computadores. & 3,42 &, 96 \\
\hline Soy bueno en el uso de software de presentación (oPowerpoint). & 3,70 &, 91 \\
\hline Soy bueno en el uso de hojas de cálculo (Excel). & 3,18 & 1,08 \\
\hline Soy capaz de configurar y administrar directorios de archivos. & 3,31 &, 96 \\
\hline “Habilidades Técnicas-Tecnológicas” & $\mathbf{3 , 6 4}$ &, $\mathbf{6 1}$ \\
\hline & & \\
\hline
\end{tabular}

Nota: De 1= totalmente en desacuerdo a 4 = totalmente de acuerdo. 
Como podemos observar, se obtuvieron altas puntuaciones respecto a lo habilidosos que se consideraban desde un punto de vista tecnológico por las tecnologías.

Las dos últimas dimensiones del cuestionario se referían directamente a la experiencia que había tenido con el software social (tabla 5) y su interés por diferentes herramientas de software social (tabla 6).

Tabla 5. La experiencia social del software

\begin{tabular}{|l|c|c|}
\hline \multicolumn{1}{|c|}{ ¿Cuál ha sido tu experiencia con... } & M & D.tp. \\
\hline los blogs? & 2,36 & 1,11 \\
\hline las wikis? & 2,28 & 1,21 \\
\hline los marcadores sociales (Delicious, Diigo)? & 1,70 & 1,04 \\
\hline $\begin{array}{l}\text { las videoconferencias realizadas a través de la web? (Elluminate, Adobe } \\
\text { Connect, Skype,...). }\end{array}$ & 2,80 & 1,30 \\
\hline las redes sociales? (Facebook, MySpace,...).. & 3,90 & 1,05 \\
\hline la publicación de fotografías? (Flicker, PICASA,...). & 3,21 & 1,30 \\
\hline el uso compartido de vídeos? (Youtube, vimedio,...) & 3,54 & 1,24 \\
\hline el podcasting? & 1,87 & 1,15 \\
\hline el software 3D de inmersión? (Second Life) & 1,97 & 1,57 \\
\hline "La experiencia social del software” & $\mathbf{2 , 6 3}$ &, $\mathbf{7 4}$ \\
\hline
\end{tabular}

Nota: De 1= Ninguna. No tengo ni idea acerca de ello a 5= Experto. Sé todo acerca de estas herramientas.

Como podemos observar por los valores medios encontrados, en pocos casos los alumnos se consideran con un conocimiento avanzado: “¿Cuál ha sido tu experiencia con las redes sociales? $(3,90)$, ¿Cuál ha sido tu experiencia con el uso compartido de vídeos? $(3,54)$ y ¿Cuál ha sido tu experiencia con la publicación de fotografías? $(3,21)$. En definitiva, las puntuaciones obtenidas indicarán un débil uso educativo por parte de los estudiantes de las diferentes herramientas de software social.

Tabla 6. Medias y desviaciones típicas en la dimensión “Cómo de interesado estás en..”

\begin{tabular}{|l|c|c|}
\hline \multicolumn{1}{|c|}{ ¿Cómo de interesado estás en... } & M & D.tp. \\
\hline $\begin{array}{l}\text { la utilización de las wikis que se utilizan en los cursos de formación que } \\
\text { realizas? }\end{array}$ & 1,44 &, 826 \\
\hline $\begin{array}{l}\text { los marcadores sociales que se emplean en los cursos de formación que } \\
\text { realizas? (De.licio.us, Diigo). }\end{array}$ & 2,63 &, 918 \\
\hline $\begin{array}{l}\text { la herramienta para la realización de videoconferencias, las cuales se } \\
\text { emplean en los cursos de formación que realizas? (Elluminate, Adobe } \\
\text { Connect,...). }\end{array}$ & 2,46 &, 931 \\
\hline
\end{tabular}




\begin{tabular}{|l|c|c|}
\hline $\begin{array}{l}\text { contar con las redes sociales que se utilizan en los cursos de formación que } \\
\text { realizas? (Facebook, MySpace,...). }\end{array}$ & $\mathbf{2 , 8 0}$ &, 936 \\
\hline $\begin{array}{l}\text { contar con herramientas de publicación de fotos que se utilizan en los cursos } \\
\text { de formación que realizas? (Flickr, Picasa). }\end{array}$ & 2,90 &, 910 \\
\hline $\begin{array}{l}\text { descargar el vídeo y las herramientas para compartir que se utilizan en los } \\
\text { cursos de formación que realizas? (YouTube, Vimedio). }\end{array}$ & 2,64 &, 963 \\
\hline $\begin{array}{l}\text { contar con herramientas de podcasting utilizados en los cursos de formación } \\
\text { que realizas? }\end{array}$ & 2,17 &, 799 \\
\hline $\begin{array}{l}\text { redes sociales tipo Twitter y Facebook utilizados en los cursos de formación } \\
\text { que realizas? }\end{array}$ & 2,68 &, 935 \\
\hline $\begin{array}{l}\text { tener herramientas tipo e- portfolio empleadas en los cursos de formación } \\
\text { que realizas? }\end{array}$ & $\mathbf{2 , 8 1}$ &, 913 \\
\hline "Social para el Aprendizaje” & $\mathbf{2 , 6 1}$ & $\mathbf{, 9 0}$ \\
\hline
\end{tabular}

Nota: De 1= Nada de nada interesado a 4 = muy interesado

Preguntados los estudiantes respecto a si estaban interesados por el uso educativo de determinado software social, obtuvimos que un gran número de estudiantes contestó que no lo sabía, destacando la videoconferencia $(f=77)$, el podcasting $(f=62)$, los marcadores sociales $(\mathrm{f}=61)$, y el $e$-portfolio $(\mathrm{f}=59)$, como los recursos sobre los que no tenían una opinión definida. Como se desprende de las puntuaciones alcanzadas, los alumnos muestran un alto interés por el uso educativo de las diferentes herramientas de software social que se le han presentado, destacando la utilización de vídeos de diferentes sitios sociales y la publicación de imágenes.

Por lo que se refiere a cómo calificaban su experiencia en trabajar en equipo, por las medias obtenidas en un intervalo de 1 a 4 , podemos decir que tendieron a considerarla positiva $(2,85)$ y que estaban muy $(3,38)$ por la colaboración con otros estudiantes en el curso.

Presentados los resultados descriptivos, a continuación, se procede a contrastar diferentes hipótesis, referidas a las posibles influencias de determinadas variables en las contestaciones ofrecidas por los sujetos:

- Ho (Hipótesis nula): No existen diferencias significativas en función del género del estudiante, su edad, haber realizado acciones formativas en Internet, acceder de forma regular a Internet, y de su experiencia en trabajar en equipo, y en las cuatro dimensiones que lo componen ("preferencias de aprendizaje", "habilidades técnicas-tecnológicas", "experiencia en software social” y "software social para el aprendizaje"), con un riesgo alfa de equivocarnos del o,05 o inferior.

- H1 (Hipótesis alternativa): Sí existen diferencias significativas en función del género del estudiante, su edad, haber realizado acciones formativas en Internet, acceder de forma regular a Internet, y de su experiencia en trabajar en equipo, y en las cuatro dimensiones que lo componen ("preferencias de aprendizaje", 
"habilidades técnicas-tecnológicas", "experiencia en software social” y "software social para el aprendizaje”), con un riesgo alfa de equivocarnos del 0,05 o inferior.

Para contrastar la hipótesis referida al género, aplicamos la $t$ de student para muestras independientes, ejecutando en primer lugar la prueba de Levene para igualdad de las varianzas (tabla 7).

Tabla 7. T de student respecto a diferencias con el género

\begin{tabular}{|l|r|r|r|r|r|}
\hline \multirow{2}{*}{} & \multicolumn{2}{|c|}{$\begin{array}{c}\text { Prueba de } \\
\text { Levene }\end{array}$} & \multicolumn{4}{|c|}{ t de student } \\
\cline { 2 - 6 } & \multicolumn{1}{|c|}{ F } & \multicolumn{1}{c|}{ Sig. } & \multicolumn{1}{c|}{ t } & \multicolumn{1}{c|}{ gl } & \multicolumn{1}{c|}{ Sig. } \\
\hline Preferencias de aprendizaje & 0,117 & 0,732 & 2,158 & 458 & \multicolumn{1}{c|}{ o,o31(*) } \\
\hline Habilidades Técnicas-Tecnológicas & 0,756 & 0,385 & 0,852 & 457 & 0,394 \\
\hline La experiencia social del software & 4,579 & 0,033 & 4,728 & 203,056 & o,ooo(**) \\
\hline Software Social para el Aprendizaje & 1,417 & 0,235 & $-0,116$ & 456 & 0,908 \\
\hline
\end{tabular}

Nota: ${ }^{*}=$ significativo al 0,$05 ;{ }^{*}=$ significativo al 0,01

Los resultados permiten rechazar la Ho con un riesgo alfa de equivocarnos de $\mathrm{p} \leq 0,05$ en la dimensión "preferencias de aprendizaje" y de p $\leq 0,01$ en la totalidad del instrumento y en la "experiencia social del software". En contraposición, no se rechazan las Ho a un nivel de significación de p $\leq 0,05$, en las "habilidades técnicas tecnológicas", y "software Social para el aprendizaje". En todos los casos en los cuales se rechazó las Ho, las puntuaciones alcanzadas por los varones fueron superiores a la de las mujeres.

Por lo que se refiere a la edad, el estadístico que utilizamos fue el ANOVA (tabla 8).

Tabla 8. ANOVA para diferencias en función de la edad

\begin{tabular}{|l|l|r|r|r|c|r|}
\hline \multicolumn{2}{|c|}{} & $\begin{array}{c}\text { Suma de } \\
\text { cuadrados }\end{array}$ & gl & $\begin{array}{c}\text { Media } \\
\text { cuadrática }\end{array}$ & \multicolumn{1}{c|}{ F } & Sig. \\
\hline \multirow{2}{*}{$\begin{array}{l}\text { Preferencias } \\
\text { de aprendizaje }\end{array}$} & Entre grupos & 0,166 & 3 & 0,055 & 0,451 & 0,716 \\
\cline { 2 - 8 } & Dentro de grupos & 55,895 & 456 & 0,123 & & \\
\cline { 2 - 8 } & Total & 56,061 & 459 & & & \\
\hline $\begin{array}{l}\text { Habilidades } \\
\text { Técnicas- } \\
\text { Tecnológicas }\end{array}$ & Entre grupos & 1,906 & 3 & 0,635 & 1,694 & 0,168 \\
\cline { 2 - 8 } & Dentro de grupos & 170,732 & 455 & 0,375 & & \\
\cline { 2 - 8 } & Total & 172,639 & 458 & & & \\
\hline
\end{tabular}




\begin{tabular}{|c|c|c|c|c|c|c|}
\hline & & $\begin{array}{l}\text { Suma de } \\
\text { cuadrados }\end{array}$ & gl & $\begin{array}{c}\text { Media } \\
\text { cuadrática }\end{array}$ & $\mathbf{F}$ & Sig. \\
\hline \multirow{3}{*}{$\begin{array}{l}\text { La experiencia } \\
\text { social del } \\
\text { software }\end{array}$} & Entre grupos & 7,880 & 3 & 2,627 & 4,922 & $0,002(* *)$ \\
\hline & Dentro de grupos & 242,831 & 455 & 0,534 & & \\
\hline & Total & 250,712 & 458 & & & \\
\hline \multirow{3}{*}{$\begin{array}{l}\text { Software } \\
\text { Social para el } \\
\text { Aprendizaje }\end{array}$} & Entre grupos & 0,621 & 3 & 0,207 & 0,334 & 0,800 \\
\hline & Dentro de grupos & 281,136 & 454 & 0,619 & & \\
\hline & Total & 281,757 & 457 & & & \\
\hline
\end{tabular}

Nota: ${ }^{* *}=$ significativo al 0,01 .

Los valores solo permiten rechazar la Ho a un nivel de significación de $\mathrm{p} \leq \mathrm{0}, \mathrm{01}$, en la dimensión "la experiencia social del software"; no así resulta para el resto de ellas, ni en la globalidad del instrumento a un nivel de significación de p $\leq 0,05$.

Para conocer entre que edades se dieron las diferencias, aplicamos la prueba de "post hoc" (Miller, 1997) para comparaciones. Indicar que solo presentamos las puntuaciones referidas a la dimensión en la cual se obtuvieron diferencias significativas, encontrándose los valores que se ofrecen en la tabla 9.

Tabla 9. Prueba "post hoc"

\begin{tabular}{|l|l|r|r|r|}
\hline & $\begin{array}{r}\text { Diferencia de } \\
\text { medias (I-J) }\end{array}$ & Error estándar & \multicolumn{1}{c|}{ Sig. } \\
\hline 17 a 20 años & 25 a 28 años &, $26128^{*}$ & 0,11547 & o,o24(*) \\
\hline & Más de 28 años &, $38441^{*}$ & 0,11170 & $\mathbf{0 , 0 0 1 ( * * )}$ \\
\hline 21 a 24 años & Más de 28 años &, $27285^{*}$ & 0,12160 & $\mathbf{0 , 0 2 5 ( * )}$ \\
\hline
\end{tabular}

Nota: ${ }^{*}=$ significativo al 0,$05 ;{ }^{* *}=$ significativo al 0,01 .

Los valores encontrados indican que las diferencias fundamentales se han dado entre el colectivo de menor edad (17 a 20 años) con el resto de edades de los encuestados.

Para analizar la significación de haber realizado acciones en Internet y acceder de forma regular en Internet, aplicamos de nuevo la $t$ de student (tabla 10). 
Tabla 10. Prueba "post hoc"

\begin{tabular}{|l|r|r|r|r|r|}
\hline \multirow{2}{*}{$\begin{array}{c}\text { ¿Has realizado acciones formativas } \\
\text { a través de Internet a lo largo de tus } \\
\text { estudios universitarios? }\end{array}$} & \multicolumn{1}{|c|}{$\begin{array}{c}\text { Prueba de } \\
\text { Levene }\end{array}$} & \multicolumn{4}{c|}{ t de student } \\
\cline { 3 - 7 } & \multicolumn{1}{c|}{ F } & \multicolumn{1}{c|}{ Sig. } & \multicolumn{1}{c|}{ t } & \multicolumn{1}{c|}{ gl } & \multicolumn{1}{c|}{ Sig. } \\
\hline Preferencias de aprendizaje & 0,050 & 0,824 & 0,627 & 458 & 0,531 \\
\hline Habilidades Técnicas-Tecnológicas & 0,451 & 0,502 & 0,825 & 457 & 0,410 \\
\hline La experiencia social del software & 0,464 & 0,496 & $-0,469$ & 457 & 0,639 \\
\hline Software Social para el Aprendizaje & 1,268 & 0,261 & 0,233 & 456 & 0,816 \\
\hline
\end{tabular}

Accedes de forma regular a

Internet con un fin educativo

(hogar, oficina, otros)?

\begin{tabular}{|l|r|r|r|r|r|}
\hline Preferencias de aprendizaje & 0,225 & 0,635 & 0,402 & 458 & 0,688 \\
\hline Habilidades Técnicas Tecnológicas & 0,067 & 0,795 & 2,737 & 457 & $\mathbf{0 , 0 0 6 ( * * )}$ \\
\hline La experiencia social del software & 0,381 & 0,537 & 1,526 & 457 & 0,128 \\
\hline Software Social para el Aprendizaje & 0,798 & 0,372 & $-0,230$ & 456 & 0,818 \\
\hline
\end{tabular}

Nota: ${ }^{*}=$ significativo al 0,$05 ;{ }^{* *}=$ significativo al o,o1.

Indicar que, en el primero de los casos, no se rechaza ninguna de las Ho formuladas. En consecuencia, se puede señalar que no existen diferencias significativas en función de si los estudiantes hubieran realizado acciones formativas a través de Internet durante sus estudios universitarios en el Total del instrumento y en las cuatro dimensiones que lo componen ("preferencias de aprendizaje", "habilidades técnicas-tecnológicas", "experiencia en software social" y "software social para el aprendizaje"), con un riesgo alfa de equivocarnos del 0,05 o inferior. Mientras que, para el caso de acceder de forma regular en Internet, solo rechazamos las Ho formuladas en la dimensión de "Habilidades Técnicas Tecnológicas" a un nivel de $\mathrm{p} \leq 0,01 \mathrm{y}$, por tanto, podemos decir que, en líneas generales, la experiencia con internet no se ha mostrado determinante.

Finalmente, se presentan los contrastes efectuados para analizar las hipótesis formuladas respecto a la significación de la experiencia de trabajar en equipo y el interés mostrado por colaborar con otros estudiantes. Para ello, se aplicó de nuevo un ANOVA (tabla 11). 
Tabla 11. ANOVA

\begin{tabular}{|c|c|c|c|c|c|c|}
\hline \multicolumn{2}{|c|}{ Experiencia de trabajar en equipo } & $\begin{array}{l}\text { Suma de } \\
\text { cuadrados }\end{array}$ & gl & $\begin{array}{l}\text { Media } \\
\text { cuadrática }\end{array}$ & $\mathrm{F}$ & Sig. \\
\hline \multirow{3}{*}{$\begin{array}{l}\text { Preferencias de } \\
\text { aprendizaje }\end{array}$} & Entre grupos & 0,678 & 3 & 0,226 & 1,847 & 0,138 \\
\hline & Dentro de grupos & 54,809 & 448 & 0,122 & & \\
\hline & Total & 55,487 & 451 & & & \\
\hline \multirow{3}{*}{$\begin{array}{l}\text { Habilidades } \\
\text { Técnicas } \\
\text {-Tecnológicas }\end{array}$} & Entre grupos & 7,015 & 3 & 2,338 & 6,339 & $\mathbf{0 , 0 0 0}(* *)$ \\
\hline & Dentro de grupos & 164,897 & 447 & 0,369 & & \\
\hline & Total & 171,912 & 450 & & & \\
\hline \multirow{3}{*}{$\begin{array}{l}\text { La experiencia } \\
\text { social del } \\
\text { software }\end{array}$} & Entre grupos & 2,420 & 3 & 0,807 & 1,487 & 0,217 \\
\hline & Dentro de grupos & 242,440 & 447 & 0,542 & & \\
\hline & Total & 244,860 & 450 & & & \\
\hline \multirow{3}{*}{$\begin{array}{l}\text { Software } \\
\text { Social para el } \\
\text { Aprendizaje }\end{array}$} & Entre grupos & 2,776 & 3 & 0,925 & 1,489 & 0,217 \\
\hline & Dentro de grupos & 277,102 & 446 & 0,621 & & \\
\hline & Total & 279,878 & 449 & & & \\
\hline \multicolumn{7}{|c|}{$\begin{array}{l}\text { Interés por colaborar con otros } \\
\text { estudiantes }\end{array}$} \\
\hline \multirow{3}{*}{$\begin{array}{l}\text { Preferencias } \\
\text { de aprendizaje }\end{array}$} & Entre grupos & 0,514 & 4 & 0,129 & 1,054 & 0,379 \\
\hline & Dentro de grupos & 55,381 & 454 & 0,122 & & \\
\hline & Total & 55,895 & 458 & & & \\
\hline \multirow{3}{*}{$\begin{array}{l}\text { Habilidades } \\
\text { Técnicas- } \\
\text { Tecnológicas }\end{array}$} & Entre grupos & 0,594 & 4 & 0,149 & 0,392 & 0,814 \\
\hline & Dentro de grupos & 171,790 & 453 & 0,379 & & \\
\hline & Total & 172,384 & 457 & & & \\
\hline \multirow{3}{*}{$\begin{array}{l}\text { La experiencia } \\
\text { social del } \\
\text { software }\end{array}$} & Entre grupos & 5,679 & 4 & 1,420 & 2,649 & $\mathbf{0 , 0 3 3}(*)$ \\
\hline & Dentro de grupos & 242,825 & 453 & 0,536 & & \\
\hline & Total & 248,504 & 457 & & & \\
\hline \multirow{3}{*}{$\begin{array}{l}\text { Software } \\
\text { Social para el } \\
\text { Aprendizaje }\end{array}$} & Entre grupos & 7,670 & 4 & 1,918 & 3,162 & $0,014(*)$ \\
\hline & Dentro de grupos & 274,080 & 452 & 0,606 & & \\
\hline & Total & 281,751 & 456 & & & \\
\hline
\end{tabular}

Nota: ${ }^{*}=$ significativo al 0,$05 ;{ }^{* *}=$ significativo al 0,01 .

Los resultados alcanzados solo permiten rechazar la Ho al nivel de significación de $\mathrm{p} \leq \mathrm{O}, \mathrm{O} 1$ en "Experiencia de trabajar en equipo", en la dimensión "Habilidades Técnicas-Tecnológicas"; mientras, en el caso del "Interés por colaborar con otros estudiantes", en las dimensiones "experiencia social del software" y "software social para el aprendizaje", a un nivel de significación de p $\leq 0,01$.

Con el objeto de conocer entre qué colectivos se daban las diferencias significativas, aplicamos de nuevo la prueba de "post hoc". En la tabla 12, se exponen los resultados obtenidos en aquellos casos donde se encontraron diferencias significativas. 
Tabla 12. Prueba "post hoc"

\begin{tabular}{|c|c|c|c|c|c|}
\hline \multicolumn{3}{|c|}{ Experiencia de trabajar en equipo } & $\begin{array}{l}\text { Diferencia } \\
\text { de medias }\end{array}$ & $\begin{array}{l}\text { Error } \\
\text { estándar }\end{array}$ & Sig. \\
\hline \multirow{2}{*}{$\begin{array}{l}\text { Habilidades } \\
\text { Técnicas } \\
\text { Tecnológicas }\end{array}$} & \multirow[b]{2}{*}{ Muy negativa } & Positivo &,$- 30066^{*}$ & 0,07633 & $0,000(* *)$ \\
\hline & & Muy positivo &,$- 33116^{*}$ & 0,08675 & $0,000(* *)$ \\
\hline \multicolumn{6}{|c|}{ Interés por colaborar con otros estudiantes } \\
\hline \multirow{3}{*}{$\begin{array}{l}\text { La experiencia } \\
\text { social del } \\
\text { software }\end{array}$} & $\begin{array}{l}\text { No muy } \\
\text { interesado }\end{array}$ & Muy interesado &,$- 31402^{*}$ & 0,13538 & $0,021(*)$ \\
\hline & Interesado & Muy interesado &,$- 19746^{*}$ & 0,08011 & $0,014(* *)$ \\
\hline & Muy interesado & No lo se &, $25241^{*}$ & 0,12599 & $0,046(*)$ \\
\hline \multirow{3}{*}{$\begin{array}{l}\text { Software } \\
\text { Social para el } \\
\text { Aprendizaje }\end{array}$} & \multirow{3}{*}{$\begin{array}{l}\text { No muy } \\
\text { interesado }\end{array}$} & Interesado &,$- 29090^{*}$ & 0,13580 & $0,033\left(^{*}\right)$ \\
\hline & & Muy interesado &,$- 44861^{*}$ & 0,14399 & $0,002(* *)$ \\
\hline & & No lo se &,$- 33738^{*}$ & 0,17156 & $0,050(*)$ \\
\hline
\end{tabular}

Nota: ${ }^{*}=$ significativo al 0,$05 ;{ }^{* *}=$ significativo al 0,01 .

Finalmente, se analizaron las posibles relaciones entre la calificación realizada respecto a "trabajar en equipo en acciones formativas" y "estar interesado en la colaboración con otros estudiantes". Y ello se realizó mediante el coeficiente de correlación de Pearson, alcanzándose un valor del 0,202, significativo al nivel de significación del $\mathrm{p} \leq 0,000$. Dicho valor permite obtener tres consideraciones para la interpretación de los datos de acuerdo con Mateo (2006): 1) que las relaciones entre las diferentes variables analizadas son significativas, por tanto, podríamos señalar que las variables están relacionadas, 2) que las relaciones son positivas y, por tanto, las variables contrastadas aumentan en la misma dirección, y 3) que los niveles de relación no son muy elevados.

\section{DISCUSIÓN Y CONCLUSIONES}

Una de las primeras conclusiones de nuestro trabajo es que Internet se está presentando como una de las tecnologías que va penetrando con más fuerza en las Universidades, y que los estudiantes la suelen utilizar constantemente para fines educativos. Al mismo tiempo, nuestros estudiantes participantes en la investigación han mostrado un verdadero interés por la utilización de las redes sociales y diferentes herramientas de los social media para su incorporación en las acciones formativas. Destaca la red social Facebook con respecto a su utilización. Estos aspectos permiten señalar que se dan las condiciones iniciales para la incorporación de estas herramientas en la enseñanza, dato que se refuerza con el hallazgo referido a que los estudiantes se perciben como muy competentes para el manejo tecnológico-técnico de las tecnologías. 
El trabajo refuerza la validez y significación del instrumento elaborado por Anderson et al. (2010) para analizar las percepciones que los estudiantes tienen respecto a las redes sociales y hacia el trabajo colaborativo. Los índices de fiabilidad alcanzados son similares a los obtenidos por diferentes autores y en distintos contextos (Anderson y otros, 2010; Cabero, 2014; Cabero y Marín, 2014).

Por otra parte, se pone de manifiesto que las actitudes que los estudiantes tienen para trabajar en grupo son altamente significativas, como lo demuestra el mostrarse en desacuerdo con la expresión: "Trabajar en un grupo conlleva obtener resultados negativos" y de acuerdo con "Me gusta que las clases se desarrollen a través del trabajo en grupo". De todas formas, las valoraciones realizadas respecto a "Prefiero trabajar de forma individual para así poder avanzar rápidamente" y "Me gusta ayudar a otras personas al trabajar en grupo", sugieren, como indican Artola, Gálvez y González (2018), que existen diferentes grupos entre los estudiantes respecto al uso de las redes sociales y el trabajo colaborativo. Ello nos lleva a reclamar la necesidad de que el docente se asegure de las percepciones que tienen los alumnos, y aplique estrategias específicas en su utilización (AbuSeileek, 2012; Jahang et al., 2012) pues, como se ha obtenido por los resultados de este estudio, el interés en trabajar con otros estudiantes determina la actitud hacia el trabajo colaborativo.

Respecto al género, no se han encontrado diferencias significativas en las habilidades técnicas-tecnológicas, lo cual refuerza la idea de que la brecha digital de género va desapareciendo (Del Prete y Cabero, 2018), aunque sí se han obtenido respecto a la experiencia con el software social, siendo los varones los que la utilizan más.

Una línea futura de investigación podría ser analizar el uso que hacen los estudiantes de las redes sociales on-line en función de los estudios que cursan.

Finalmente, cabe subrayar que son los alumnos de menor edad quienes muestran actitudes más significativas para incorporar las redes sociales a la formación y la utilización de metodologías apoyadas en el trabajo colaborativo.

\section{REFERENCIAS}

Abuseileek, A. F. (2012). The effect of computer-assisted cooperative learning methods and group size on the EFL learners' achievement in communication skills. Computer \& Education, 58, 35-49.

Alter, A. (2018). Irresistible. ¿Quién nos ha convertido en yonquis tecnológicos? Barcelona: Paidós.

Anderson, T., Poellhuber, B., \& Mckerlich, R. (2010). Social Software survey iused with unpaced undergrad. Recuperado de $\quad$ http://auspace.athabascau.ca/ handle/2149/2771

Artola, A., Gálvez, C., y González, A. (2018). Explorando el potencial de los dispositivos electrónicos y de las redes sociales en el proceso. Edutec. Revista Electrónica de Tecnología Educativa, 64, Recuperado de http://www.edutec.es/revista

Baleghizadeh, S. (2012). Comparing traditional with cooperative pairs: the case of Iranian EEGNP students. Procedia 
Social and Behavioral Sciences, 66, 330336.

Bisquerra, R. (Coord.) (2004). Metodología de la investigación educativa. Madrid: La Muralla.

Cabero, J. (Dir.) (2014). Las redes sociales como instrumentos para la formación, Sevilla: GID.

Cabero, J., y Marín, V. (2014). Posibilidades educativas de las redes sociales y el trabajo en grupo: percepciones de los alumnos universitarios. Comunicar, 42, 165-172.

Cabero, J., Barroso, J., Llorente, M. C., y Yañez, C. (2016). Redes sociales y Tecnologías de la Información y la Comunicación en Educación: aprendizaje colaborativo, diferencias de género, edad y preferencias. RED. Revista de Educación a Distancia. 51(1). Recuperado de http:// www.um.es/ead/red/cabero et al.pdf

Chávez, I., y Gutiérrez, M. C. (2015). Redes sociales como facilitadoras del aprendizaje de ciencias exactas en la educación superior. Apertura, 7(2), 1-12.

Cruz, I. (2016). Percepciones en el uso de las redes sociales y su aplicación en la enseñanza de las matemáticas. Pixel-Bit. Revista de Medios y Educación, 48, 165186.

De Haro, J. (2011). Redes sociales para la educación. Madrid: Anaya.

Del Prete A. C., y Cabero, J. (2018). Digital Gender Gap among Teachers: An Issue Pending Resolution (A Research Study at INACAP, Chile.

Della-Porta, D. (2015). Social Movements in Times of Austerity: Bringing Capitalism Back into Protest Analysis. Cambridge: Polity Press.

Delgado-García, M., García-Prieto, F. J., y Gómez-Hurtado, I. (2018). Moodle y Facebook como herramientas virtuales didácticas de mediación de aprendizajes: opinión de profesores y alumnos universitarios. Revista Complutense de Educación, 29(3), 35-52.
Fromet, F., García, A., y Bohórquez, M. R. (2017). The Use of Social Networks as a Communication Tool between Teachers and Students: A Literature Review. TOJET: The Turkish Online Journal of Educational Technology, 16(4), 126-144.

García, R., Tirado, R., y Hernando, A. (2018). Redes sociales y estudiantes: motivos de uso y gratificaciones. Evidencias para el aprendizaje. Aula Abierta, 47(3), 291298.

Gavilán, D., Martínez, G., y Fernández, S. (2017). Universitarios y redes sociales informativas: Escépticos totales, moderados duales o pro-digitales. Comunicar, $X X V(53), 61-70$.

Jahng, N., Nielsen, W.S., \& Chan, E.K. (2010). Collaborative learning in an online course: A comparison of communication patt erns in small and whole group activities.

$\mathrm{Ku}, \mathrm{H}-\mathrm{Y}$., Tseng, H-W., y Akarasriwo, Ch. (2013). Collaboration factors, teamwork satisfaction and student attitudes toward online collaborative learning. Computers in human Behavior, 29, 922-929.

Levis, D. (2011). Redes educativas 2.1. Medios sociales, entornos colaborativos y procesos de enseñanza y aprendizaje. Revista de Universidad y Sociedad del Conocimiento (RUSC), 8(1), 7-24.

López, M. C., Flores, K., Espinoza, A., y Rojo, D. (2017). Posibilidades de Facebook en la docencia universitaria desde un caso de estudio. Apertura, 9(2), 132-147. doi: http://dx.doi.org/10.18381/Ap.v9n2.1133

Mateo, J. (2006). La investigación ex post-facto. En R. Bisquerra (Coord.), Metodología de la investigación educativa. Madrid: La Muralla, 195-230.

Miller, J. (1997). Beyond ANOVA: Basics of applied statistics. London: CRC Press.

Romero, A., y Garay, U. (2017). Aprendizaje colaborativo a través de redes sociales en contextos universitarios. Edutec. Revista Electrónica de Tecnología Educativa, 62. Recuperado de http://www.edutec.es/ revista 
O’ Dwyer, L. y Bernauer, J. (2014). Quantitative research for the quialitative researcher. London: Sabe.

Sabariego, M. (2012). El proceso de investigación (parte 2). En R. Bisquerra (Coord.), Metodología de la investigación educativa (127-163). ( ${ }^{\mathrm{a}}$. ed.). Madrid: La Muralla.

Sanz, E., Alonso, R. A., Sáenz, M., Ponce, A., y Valdemoros, M. A. (2018). Ocio, redes sociales y estudiantes españoles.
Educación $X X 1,21(2), \quad 59-78$, doi: 10.5944/educXX1.19538.

Tejada, E., Garay, U., y Romero, A. (2017). La incidencia de la participación cooperativa en entornos virtuales en el rendimiento académico. Edutec. Revista Electrónica de Tecnología Educativa, 6o. Recuperado de http://www.edutec.es/revista

Vázquez, A. I., y Cabero, J. (2015). Las redes sociales aplicadas a la formación. Revista Complutense de Educación, 26, 253-272.

\section{PERFIL ACADÉMICO Y PROFESIONAL DE LOS AUTORES}

Julio Cabero Almenara. Catedrático de Tecnología Educativa de la Universidad de Sevilla (España), director del Secretariado de Recursos Audiovisuales y Nuevas Tecnologías de la Universidad de Sevilla, ha impartido cursos y conferencias en diferentes universidades españolas, europeas y latinoamericanas. Miembro fundador de la Asociación para el desarrollo de la Tecnología Educativa (EDUTEC). E-mail: cabero@us.es

Dirección:

Universidad de Sevilla c/ Jiménez Aranda 19, 7, $4^{\circ}$ A 41018 Sevilla. España

Annachiara Del Prete. Asesora pedagógica por el Centro de Innovación en Investigación CIEDU por la Universidad Tecnológica de Chile INACAP Chile. Licenciada en Literatura Moderna con especialidad en Historia Contemporánea por la Universidad Federico II de Nápoles (2000). Doctora en Tecnología Educativa por la Universidad Rovira y Virgili de Tarragona, España (2010). Máster Europeo en "Políticas locales, género y desarrollo" (2007) por la Universidad Jaume I. Máster en Comunicación y Tecnología Multimedia por el COREP de Turín, Italia (2003).

E-mail: aadelprete@inacap.cl

María Luisa Arancibia Muñoz. Asesora pedagógica por el Centro de Innovación en Investigación CIEDU por la Universidad Tecnológica de Chile INACAP Chile. Socióloga de la Universidad de Concepción, Chile. Magister en Políticas Sociales, Doctorado (c) en Ciencias Sociales y Jurídicas de la Universidad de Córdoba, España.

E-mail: marancibiam@inacap.cl 
Dirección:

INACAP Casa Central, Avenida del Cóndor 720,

Ciudad Empresarial, Santiago. Chile

Fecha de recepción del artículo: 04/10/2018

Fecha de aceptación del artículo: 23/01/2019

Fecha de aprobación para maquetación: 17/02/2019 